LA-UR-01-2411

Approved for public release; distribution is unlimited.

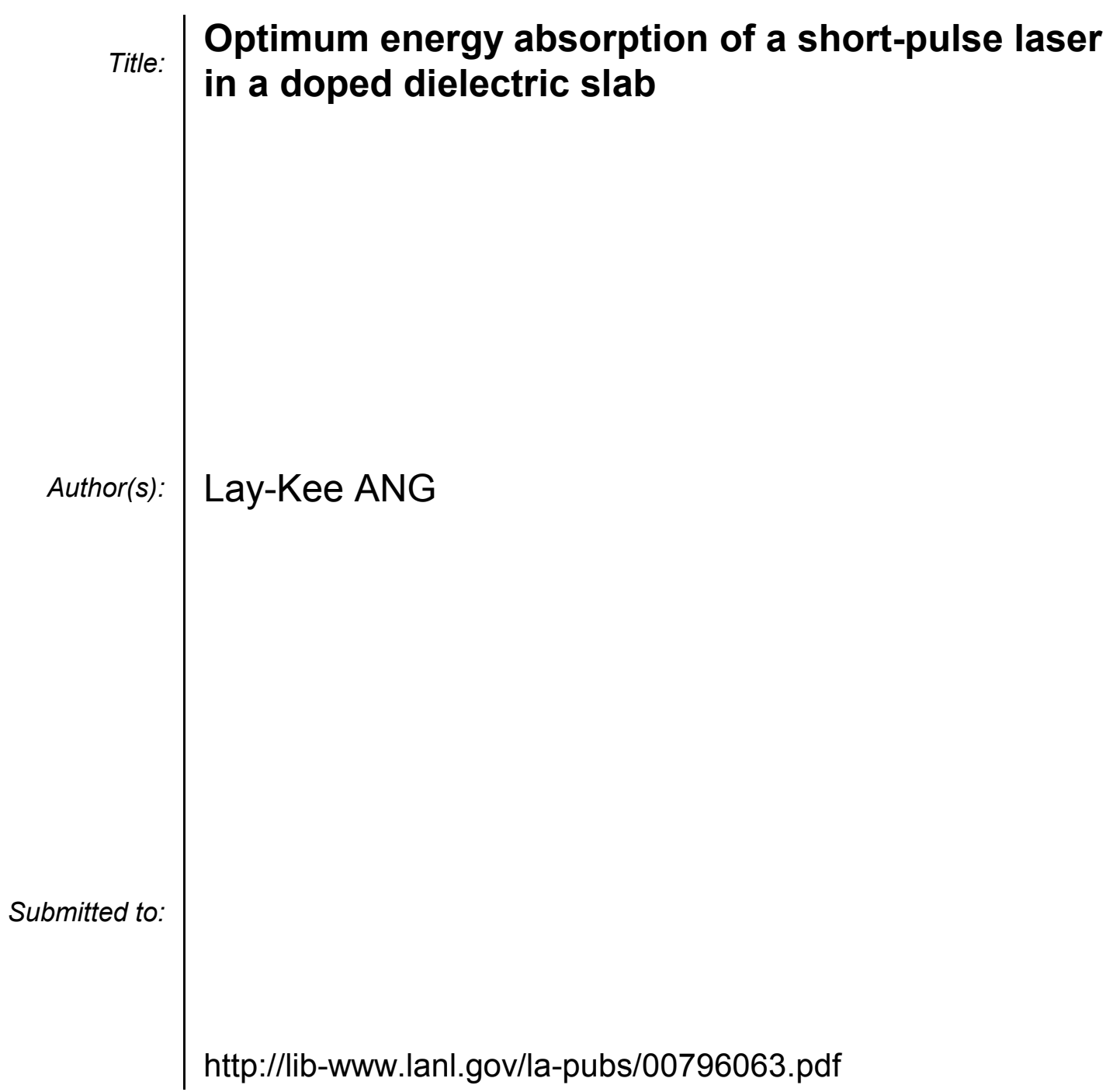

Los Alamos National Laboratory, an affirmative action/equal opportunity employer, is operated by the University of California for the U.S. Department of Energy under contract W-7405-ENG-36. By acceptance of this article, the publisher recognizes that the U.S. Government retains a nonexclusive, royaltyfree license to publish or reproduce the published form of this contribution, or to allow others to do so, for U.S. Government purposes. Los Alamos National Laboratory requests that the publisher identify this article as work performed under the auspices of the U.S. Department of Energy. Los Alamos National Laboratory strongly supports academic freedom and a researcher's right to publish; as an institution, however, the Laboratory does not endorse the viewpoint of a publication or guarantee its technical correctness. 


\title{
Optimum energy absorption of a short-pulse laser in a doped dielectric slab
}

\author{
Lay-Kee ANG \\ Applied Physics Division, Los Alamos National Laboratory, \\ Los Alamos, NM 87545, USA \\ E-mail: lkang@lanl.gov
}

\begin{abstract}
A model is used to calculate energy absorption efficiency when a short-pulse laser impinges on a dielectric slab doped with an impurity for which the electrons have a resonant line at the laser wavelength. The amount of the energy resonant absorption is due to the overlapping between laser spectrum and resonance spectrum. The energy absorption efficiency can be maximized for a certain degree of doping concentration (at a given pulselength) and also for a certain pulselength (at a given doping concentration). For a modest amount of impurity, the resonant absorption may increase the fraction of energy absorption up to tens of percent of laser energy at 100s optical cycles when the laser wavelength is tuned within $1 \%$ of the resonant line. Dimensionless parameters are constructed so that the scaling to various parameters: laser wavelength, laser pulselength, dielectric constant, slab thickness, impurity concentration, resonant linewidth, and separation between the laser wavelength and the line resonance, could easily be obtained.
\end{abstract}

Keywords: resonant absorption, doped dielectric, ultrafast laser

\section{Introduction}

In laser-dielectric interaction, it is crucial to assess the amount of laser energy that is absorbed especially when the dielectric is nearly transparent to the light. The amount of energy absorbed is important to many applications, such as laser micro-machining, optical lithography, charge particle acceleration, laser-induced dielectric breakdown, and ophthalmological procedures [1-4]. Recently, short laser pulses on the order of 100s of optical cycles become available from chirped pulse lasers [5], and modern free electron lasers [6]. For example, a chirped-pulse amplified Ti:sapphire laser system $(780 \mathrm{~nm}, 80-500 \mathrm{fs})$ at the Center for Ultrafast Optical Science, University of Michigan, has about 30 - 200 optical cycles [1]. The Thomas Jefferson laboratory's free electron laser system $(3-6.6 \mu \mathrm{m}, 1 \mathrm{ps}, 1$ $\mathrm{kW})$ has about 50 - 100 optical cycles [6]. The free electron laser is highly tunable by the energy of the electron beam, while the chirped pulse laser offers considerable flexibility in adjusting the pulselength.

We are particularly interested in the enhanced energy absorption when the dielectric is doped with an impurity having a resonance line at the laser wavelength. The amount of energy absorption depends on laser wavelength, laser pulselength, resonance linewidth, impurity concentration, material properties, slab thickness, and degree of resonance. The amount of resonant energy absorption had been calculated [7]. In this paper, we present the calculation of energy transmission, reflection, and absorption in a doped dielectric slab as a function of laser pulselength and impurity concentration. The results for small off-resonance conditions will also be given.

\section{Analysis}

In this model, we assume a lossless dielectric slab with a thickness $L$ has a relative dielectric constant $\varepsilon$ when impurity is absent. The amount of energy absorbed in the dielectric is determined by the amount of impurity, which contributes an imaginary part to the refractive index. We use the Drude model to account for the impurity number density $\mathrm{N}$ with a corresponding electron plasma frequency $\omega_{\mathrm{p}}$. Numerically, $\omega_{\mathrm{p}}[\mathrm{THz}]=2 \pi \times 9 \times\left[\mathrm{N} / 10^{18} \mathrm{~cm}^{-3}\right]^{1 / 2}$. The impurity is assumed to have a resonant frequency $\omega_{0}$ and a finite linewidth $\omega_{0} / Q$, where we have used the quality factor $\mathrm{Q}$ whose inverse represents the width of the resonance. In the model, the refractive index (n) takes on the usual form:

$$
n^{2}=\varepsilon-\frac{\omega_{p}^{2}}{\omega^{2}-j \omega \omega_{0} / Q-\omega_{0}^{2}},
$$

where $\omega \approx \omega_{\mathrm{o}}$ is the laser frequency. 
Consider that the energy content of the incident laser pulse is unity. From energy conservation, the energy absorption in the dielectric slab equals to the incident wave energy minus the reflected wave energy and the transmitted wave energy. Using a transmission line model and Parseval's theorem, we can convert the energy content representation of the wave in time domain to frequency domain. For a laser pulse with a Gaussian shape in time, the energy absorption efficiency $A$ is

$$
A=\frac{\tau}{\sqrt{2 \pi}} \int_{-\infty}^{\infty} d \omega e^{-\left(\omega-\omega_{E}\right)^{2} \tau^{2} / 2}\left[1-|R|^{2}-|T|^{2}\right],
$$

where $\omega_{\mathrm{E}}$ is the laser center frequency, and $\tau$ is the laser pulselength. Here, $\mathrm{R}$ and $\mathrm{T}$ are the reflection coefficient and transmission coefficient (in complex amplitude) respectively, which are a function of $\mathrm{n}$ and $\omega \mathrm{L}$ [7].

It is obvious that the amount of resonant absorption depends on the sum of overlapping between the laser spectrum and the resonance spectrum of the impurity in the frequency domain. From Eqs. (1) and (2), we see that the resonance spectrum has a linewidth of $\omega_{0} / \mathrm{Q}$ centering at $\omega_{\mathrm{o}}$, and the laser spectrum has a linewidth of $2 / \tau$ centering at $\omega_{\mathrm{E}}$. As $\mathrm{Q} \rightarrow \infty$, the width of the resonance spectrum becomes small and most of the laser spectrum will not see the resonance spectrum, which implies no energy absorption as $Q \rightarrow \infty$. It is also easy to see that the overlapping can be optimized at some laser pulsewidth $\tau$, as the laser spectrum amplitude increases with $\tau$ and its linewidth decreases with $\tau$ for the same energy content in the laser pulse. Thus for a giving resonance spectrum, we could vary the laser pulsewidth to obtain an optimal energy absorption efficiency $A$ (see Fig. 1).

For convenience of scaling, we introduce a set of normalized parameters:

$$
\delta=\frac{\omega_{p}^{2}}{2 \varepsilon \omega_{0}^{2}}, \Lambda=\frac{\omega_{0} \tau \delta}{\sqrt{2}}, \rho=\frac{\omega_{E}-\omega_{0}}{\omega_{0} \delta}, \kappa=\frac{L}{\lambda \sqrt{\varepsilon}}
$$

where $\lambda=2 \pi \mathrm{c} / \omega_{0}$ is the free space wavelength. Here, $\delta$ measures the doping density, $\Lambda$ measures the laser pulselength for a fixed $\delta, \rho$ measures the separation between the laser frequency $\omega_{\mathrm{E}}$ and the dielectric dopant transition frequency $\omega_{0}$, and $\kappa$ measures the width of the dielectric slab. In terms of the dimensionless parameters, Eqs. (1) and (2) become

$$
n=\sqrt{\varepsilon} \sqrt{1-\frac{1}{y-j /(2 Q \delta)}},
$$

and

$$
A=\frac{\Lambda}{\sqrt{\pi}} \int_{-\infty}^{\infty} d y e^{-\Lambda^{2}(y-\rho)^{2}}\left[1-|R|^{2}-|T|^{2}\right],
$$

at $\omega_{\mathrm{E}} \approx \omega_{0}$, where $\mathrm{R}$ and $\mathrm{T}$ depends on $\varepsilon, \mathrm{Q} \delta, \kappa$ and $\mathrm{y}=(\omega-$ $\left.\omega_{0}\right) /\left(\omega_{0} \delta\right)$ is a dummy variable for the integration. Here, Eq. (5) gives the energy absorption efficiency $A$ as a function of five parameters: $\varepsilon, \mathrm{Q} \delta, \kappa, \Lambda, \rho$. Note $A=0$ for a lossless dielectric when the impurity is absent $(\delta=0)$.

It is interesting that the resonant linewidth $\mathrm{Q}$ and the concentration of the impurity $\delta$ enter in the product $\mathrm{Q} \delta$ in Eq. (4). As we have remarked before, there can hardly be any absorption if $\mathrm{Q}$ becomes infinity or $\delta$ becomes zero. Thus, $A$ increases from zero, reaches some maximum value, and decreases back to zero as the product $\mathrm{Q} \delta$ increases from zero to infinity. The last statement implies that there is an optimal doping to yield maximum energy absorption efficiency for a given laser pulselength.

\section{Resonance}

We first show the results when the laser frequency is at exact resonance, $\omega_{\mathrm{E}}=\omega_{0}(\rho=0)$. Shown in Fig. 1 is the energy absorption efficiency $A$ as a function of $\Lambda$ for various $\mathrm{Q} \delta=5$ to $5 \times 10^{6}$ at $\varepsilon=4$ and $\mathrm{L} / \lambda=1000$.

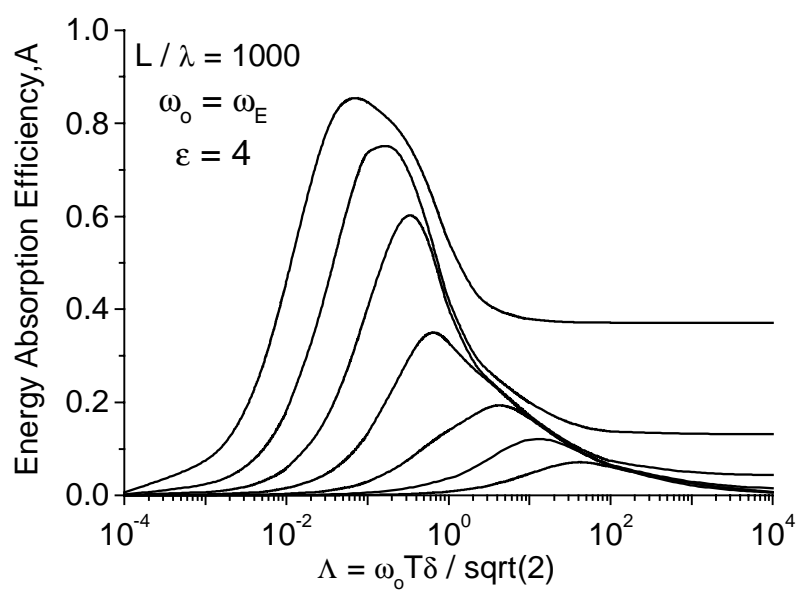

Fig. 1 Energy absorption efficiency A as a function of $\Lambda$ for various $\mathrm{Q} \delta=5$ to $5 \times 10^{6}$ (top to bottom) at exact resonance $\omega_{\mathrm{E}}=\omega_{\mathrm{o}}(\rho=0), \varepsilon=4$ and $\mathrm{L} / \lambda=1000$.

For a given $\mathrm{Q} \delta$, there is an optimal $\Lambda$ for maximum energy absorption. For a given $\mathrm{Q}$, this finding implies that the energy absorption efficiency can be maximized for a certain degree of doping concentration $\delta$ (at a given 
pulselength $\tau$ ) and also for a certain pulselength $\tau$ (at a given doping concentration $\delta$ ). For a modest amount of impurities, the resonant absorption may increase the absorption up to tens of percent of laser energy at 100s of laser optical periods $\mathrm{f} \tau$. As an example, consider a laser wavelength of $248 \mathrm{~nm}$, a resonance width of $0.005 \mathrm{~nm}$, and impurity concentration of $\delta=10^{-4}\left(\mathrm{~N}=1.43 \times 10^{17} \mathrm{~cm}^{-3}\right)$, we have $\mathrm{Q} \delta=5$, and energy absorption efficiency becomes maximum $A \approx 85 \%$ at a laser pulselength of about $83 \mathrm{fs,}$ which is equivalent to about 100 laser optical periods (see Fig. 2 below).

In Fig.2, we show the dependence of the laser energy absorption, transmission and reflection on the number of laser optical periods at a fixed $\mathrm{Q} \delta=5$ and $\delta=10^{-4}$. It shows that the distribution of the energy may be adjusted by varying the laser pulselength, where most of energy is reflected for a long laser pulse at $\mathrm{f} \tau>>10^{4}$.

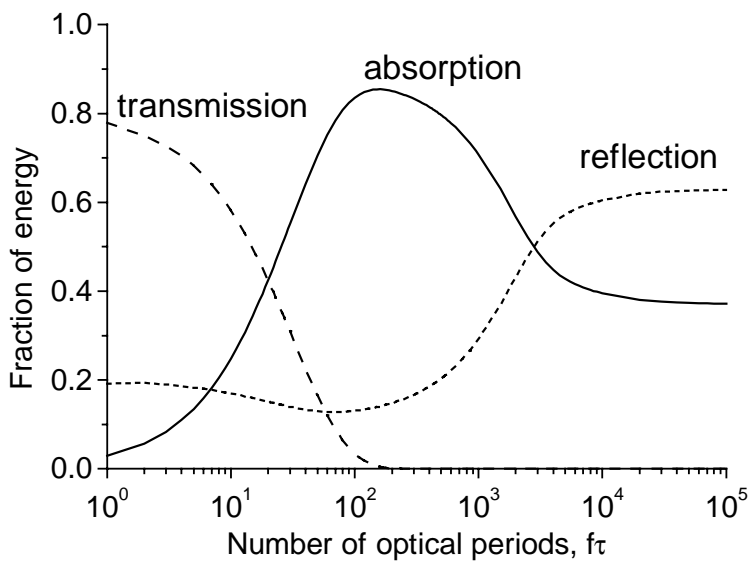

Fig. 2 Fraction of energy absorption, transmission, and reflection as a function of laser pulselength $(f \tau)$ in units of optical periods for $\varepsilon=4, \rho=0, \mathrm{Q} \delta=5, \delta=$ $10^{-4}$ and $\mathrm{L} / \lambda=1000$

In Fig. 3, we plot the fraction of energy absorption, transmission, and reflection as a function of impurity density $\delta$ at a fixed laser pulselength. Here we have set $\varepsilon=$ 3.3, $\rho=0, Q=5 \times 10^{4}, \tau=100 \mathrm{fs}, \mathrm{L}=5.7 \mu \mathrm{m}, \lambda=248 \mathrm{~nm}$, and varied the $\delta$ from $10^{-6}$ to $10^{-2}$, which corresponds to $\mathrm{N}=$ $1.17 \times 10^{17} \mathrm{~cm}^{-3}$ to $1.17 \times 10^{21} \mathrm{~cm}^{-3}$. Figure 3 shows that the ratio of the energy distribution could be adjusted over a wide range of doping density. If the doping density becomes very high, the refractive index becomes almost imaginary, in which case little laser energy could be absorbed. Thus, at a sufficiently high doping density (beyond the optimal density $\delta \approx 5 \times 10^{-4}$ at $A \approx 0.5$ ), the doped dielectric behaves more like a reactive medium, where most of the energy is reflected. Additional impurities will only increase the energy reflected from the dielectric. On the other hand, most of the energy is transmitted as expected when $\delta$ is small.

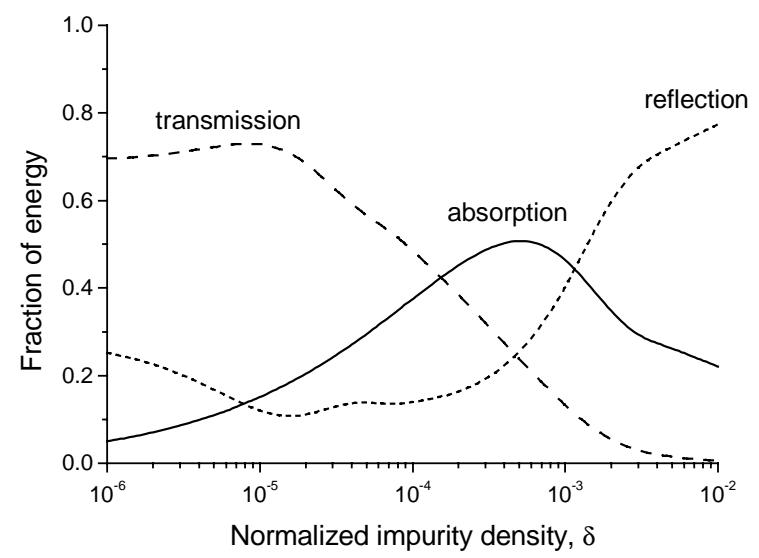

Fig. 3 Efficiency of energy absorption, transmission and reflection as a function of the normalized doping density $(\delta)$ for $\varepsilon=3.3, \rho=0, \mathrm{Q}=5 \times 10^{4}, \mathrm{f} \tau=120$, and $\mathrm{L} / \lambda=15$.

\section{Off-Resonance}

In this session, we will study the effects of small offresonance from the resonant line $\left(\omega_{\mathrm{E}} \neq \omega_{\mathrm{o}}\right)$ on the energy absorption efficiency $A$. First, we define $\alpha \equiv\left(\omega_{\mathrm{E}}-\omega_{\mathrm{o}}\right) / \omega_{\mathrm{o}}$ to measure the percentage of off-resonance from resonant frequency $\omega_{\mathrm{o}}$. In Fig. $4, A$ is plotted as a function of the laser pulselength (in units of optical periods) for various values of $\alpha= \pm 10^{-6}$ to $\pm 10^{-2}$ for a set of parameters: $\varepsilon=4$, Q $=5 \times 10^{4}, \delta=10^{-4}$, and $\mathrm{L} / \lambda=1000$.

As discussed before, the energy absorption efficiency $A$ depends on the sum of overlapping between the resonance spectrum and laser spectrum. For convenience, let's define the energy absorption efficiency at exact resonance $(\alpha=0)$ as $A_{o}$ (solid line in Fig. 4) and the normalized resonance width as $\mathrm{Q}^{-1}$ (i.e. $\mathrm{Q}=5 \times 10^{4}$, we have $\mathrm{Q}^{-1}=2 \times 10^{-5}$ in this case). If the laser spectrum lies within the resonance spectrum, such that $\alpha<\mathrm{Q}^{-1}, A$ converges to $A_{o}$ as indicated by $\alpha= \pm 10^{-6}$ and $= \pm 10^{-5}$ in Fig. 4 . On the other hand, if the separation of the two spectra is much larger than $\mathrm{Q}^{-1}, A(<$ $0.3)$ decreases from $A_{o}$ as shown in the case $\alpha= \pm 10^{-2}$. In between the two limits, such as $\pm 10^{-3}$, the behavior of $A$ is more complicated depending on the location of the laser spectrum $G(y)=\Lambda \exp \left(-\Lambda^{2}(y-\rho)^{2}\right)$ with respect to the single- 
frequency absorption coefficient, $\quad \mathrm{F}(\mathrm{y})=1-|R|^{2}-|T|^{2}$ as shown in Fig. 5 below.

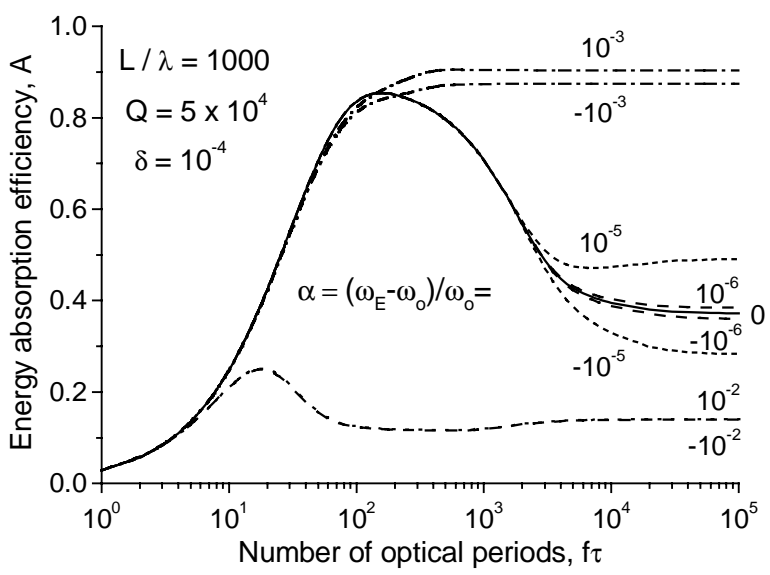

Fig. 4 Energy absorption efficiency $(A)$ as a function of the number of optical periods (f $\tau$ ) expressed at various off-resonance, $\alpha=\left(\omega_{\mathrm{E}}-\omega_{\mathrm{o}}\right) / \omega_{\mathrm{o}}$. The value of $\alpha=0$ (solid line), $\pm 10^{-6}, \pm 10^{-5}, \pm 10^{-3}$ and $\pm 10^{-2}$ (dash lines).

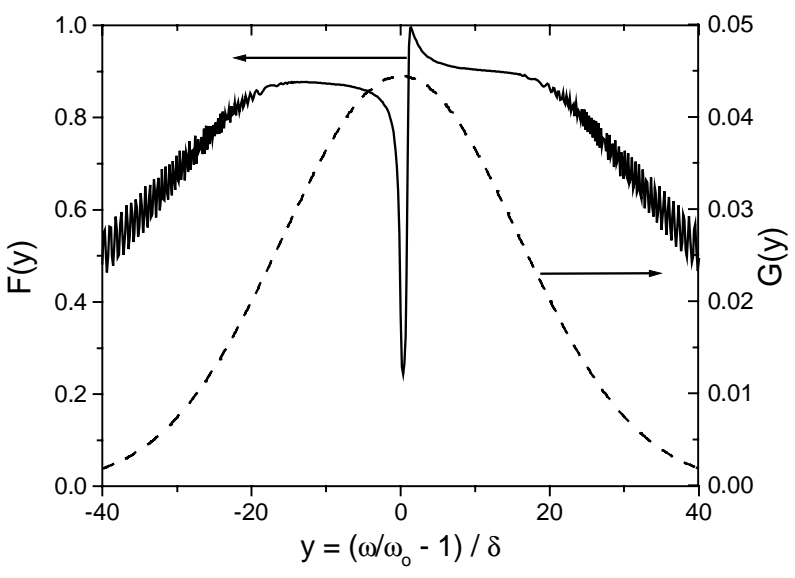

Fig. 5 The single-frequency absorption coefficient $\mathrm{F}(\mathrm{y})=$ $1-|R|^{2}-|T|^{2}$, and laser spectrum $\mathrm{G}(\mathrm{y})=\Lambda \exp (-$ $\left.\Lambda^{2}(\mathrm{y}-\rho)^{2}\right)$ as a function of y for $\varepsilon=4, \mathrm{Q}=5 \times 10^{4}, \delta=$ $10^{-4}$, $\mathrm{f} \tau=100$, and $\mathrm{L} / \lambda=1000$. At $\mathrm{y}=0, \mathrm{~F}(0)=$ 0.372 . For reference, $G(y)$ is plotted at $\rho=0$.

Figure 5 shows that $\mathrm{F}(\mathrm{y})$ has a steep region $(-1.44<\mathrm{y}<$ 1.44), and a flat region ( $\mathrm{y}>1.44$ and $\mathrm{y}<-1.44)$. If the laser spectrum $\mathrm{G}(\mathrm{y})$ is detuned considerably that it lies mostly in the flat region, $A$ could be larger than $A_{o}$, as shown in the case $\alpha= \pm 10^{-3}$ in Fig. 4 . In the figure, we see that $\mathrm{F}(\mathrm{y})$ is asymmetry close to $\mathrm{y}=0$, which explains why the energy absorption for $\alpha>0$ is higher than that for $\alpha<0$ for same magnitude of $\alpha$. This asymmetry in energy absorption comes from the fact that the refractive index, $n$, is not symmetric about $\omega \approx \omega_{\mathrm{o}}$ when the impurity concentration is high, as in the case of a solid.

\section{Summary}

We have used a simple model to estimate the enhanced energy absorption of a short laser pulse in a doped dielectric slab. It is quite remarkable that even for laser frequency at rather large deviation of $1 \%$ from the resonant line, $10 \%$ of laser energy may still be absorbed (Fig. 4). The applicability of this simple model of resonant absorption may be tested with a tunable laser whose frequency can be adjusted to coincide with the resonance line of the impurities embedded in the dielectric. Indeed, by varying the dopant concentration or the laser pulselength, one might even infer the intrinsic linewidth from the measured absorption efficiencies. On a much more modest scale, it was found experimentally that, when a copper vapor laser (510 and $578 \mathrm{~nm}$ ) shines on a transparent dielectric (polymethylmethacrylate - Lucite), the light becomes strongly absorbed if the dielectric surface is painted with ink (propynol based solvent Color Index blue 70) [8]. One may well wonder whether such greatly enhanced absorption is of the resonant type studied in this paper. Finally we should point out that this model may also be applicable to gas-phase absorption by short pulse laser or even microwave; an example of the microwave source would be a $100 \mathrm{~ns}$ microwave pulse at $1.3 \mathrm{GHz}$, which is equivalent to 130 cycles [9].

\section{References}

[1] X. Liu, D. Du, and G. Mourou, IEEE J. Quantum Elect. 33, 1706 (1997).

[2] J. Canning, J. Vac. Sci. Technol. B 15, 2109 (1997).

[3] E. Esarey, P. Sprangle, J. Krall, and A. Ting, IEEE Trans. Plasma Sci. 24, 252 (1996).

[4] R. M. Kurtz, X. B. Liu, V. M. Elner, J. A. Squier, D. T. Du,and G. A. Mourou, J. Refrac. Surg. 12, 653 (1997).

[5] M. D. Perry and G. Mourou, Science 264, 917 (1994).

[6] G. R. Neil and Jefferson Lab. FEL Team, Nucl. Instrum. Meth. B 144, 40 (1998).

[7] L. K. Ang, Y. Y. Lau, and R. M. Gilgenbach, Appl. Phys. Lett. 74, 2912 (1999).

[8] P. L. G. Ventzek, R. M. Gilgenbach, C. H. Ching, and R. A. Lindley, J. Appl. Phys. 72, 1696 (1992).

[9] K. J. Hendricks, P. D. Coleman, R.W. Lemke, M.J. Arman, and L. Bowers, Phys. Rev. Lett. 76, 154 (1996). 